\title{
Comparison of OnBaseU Tests with Biomechanical Motion Analysis in Youth Baseball Pitchers
}

\author{
Tessa Hulburt, MS ${ }^{1}$ (D) ${ }^{\text {, }}$, Taylor Catalano, BS ${ }^{2}$, Kristen F. Nicholson, PhD ${ }^{1}$ (D) \\ ${ }^{1}$ Orthopaedic Surgery, Wake Forest School of Medicine, ${ }^{2}$ Wake Forest School of Medicine \\ Keywords: baseball, motion analysis, onbaseu, pitching, movement screen \\ https://doi.org/10.26603/001c.29519
}

\section{International Journal of Sports Physical Therapy}

Vol. 16, Issue 6, 2021

\section{Background}

The OnBaseU screen was developed to evaluate a baseball pitcher's ability to perform movement patterns key to pitching. However, due to lack of validation, it is unclear what application is ideal for this screen.

\section{Purpose}

To compare four OnBaseU tests to relevant pitching mechanics measured using 3D motion capture to evaluate if the OnBaseU screen can be used to assess pitching mechanics. The secondary purpose was to compare OnBaseU and 3D motion capture seated trunk rotation test results to determine the validity of the OnBaseU test.

\section{Methods}

OnBaseU screening and 3D motion capture pitching evaluations were completed for 103 adolescent pitchers (age $=15.2 \pm 1.29$ years; height $=1.80 \pm 0.0866 \mathrm{~m}$; weight $=76.2 \pm 13.8$ $\mathrm{kg})$. A motion capture seated trunk rotation test was also conducted on 80 of the 103 youth players $($ age $=15.2 \pm 1.32$ years; height $=1.80 \pm 0.0889 \mathrm{~m}$; weight $=75.7 \pm 13.9 \mathrm{~kg}$ ).

\section{Results}

Stride length and OnBaseU side step walkout test data were moderately correlated, and all other comparisons were not correlated or were minorly correlated. No significant differences were found between kinematics from players who obtained different OnBaseU scores, except for stride lengths during pitching of players who scored a 1 or 3 on the OnBaseU side step walkout test $(\mathrm{p}<0.01)$. Further, OnBaseU and motion capture seated trunk rotation tests were not correlated $(r=0.003)$ and not found to be statistically associated $(\mathrm{p}=0.83)$.

\section{Conclusion}

Results from this study indicate that the OnBaseU clinical assessment screen may not have use in assessing pitching mechanics and that visual grading criteria used in the OnBaseU seated trunk rotation test may not be accurate.

\section{Level of Evidence}

3

\section{INTRODUCTION}

Despite increased knowledge regarding pitching mechanics and the implementation of pitch count limitations, the in- cidence of injury among youth baseball pitchers is still on the rise. ${ }^{1,2}$ Improper pitching mechanics has been identified to play a large role in injury risk. ${ }^{3-6}$ Increased elbow valgus torque, decreased shoulder rotational range of mo-

\footnotetext{
a Corresponding Author:

Tessa Hulburt,

Medical Center Boulevard, Winston-Salem, NC 27157-1070

p 336-716-1787

thulburt@wakehealth.edu
} 
tion, knee flexion at front foot contact, and early rotation of the hips and shoulders have all been found to correlate with increased risk of injury for baseball pitchers. ${ }^{4-6}$ Evaluation of pitching mechanics is integral for injury prevention as well as for performance improvement.

The existing gold standard for pitching performance and injury prevention analysis is evaluation with a 3D motion capture system. However, most athletes do not have access to these systems due to their cost, limited availability, and the need for a team biomechanist to operate the system, process data, and assist with interpretation of findings. Validated clinical movement screens have previously been utilized to estimate an athlete's risk of injury as well as their overall performance. The Golf Movement Screen (GMS) evaluates a golfer's movement ability in 10 different exercises. Through biomechanical analysis using an electromagnetic tracking system, the GMS has been shown to correlate with important aspects of golf swing mechanics, such as spine control and increased separation between the upper torso and pelvis rotation. ${ }^{7}$ The Functional Movement Screen $\left(\right.$ FMS $^{\mathrm{TM}}$ ) is a widely used measurement of movement ability that has also been shown to be predictive of musculoskeletal injury. ${ }^{8,9}$ The FMS ${ }^{\mathrm{TM}}$ measures dynamic balance, strength, and flexibility, ${ }^{10}$ and a lower FMS ${ }^{\mathrm{TM}}$ score suggests improper fundamental movement patterns, and increased injury risk. ${ }^{11}$ This concept has been validated in multiple studies assessing the correlation between FMS ${ }^{\text {тM }}$ score and serious injury. $8,12,13$

The OnBaseU screen is a clinical assessment tool developed by a team of baseball and softball coaching, strength and conditioning, biomechanics, and medical experts. OnBaseU University ${ }^{\circledR}$ is a for profit organization that requires a certification course to administer the screening assessment. No educational requirements are necessary to obtain certification. Developers state that the screen is meant to assess the movement patterns of baseball and softball pitchers and hitters through 16 movement tests. ${ }^{14}$ Any rationale for or validity of each component of the movement screen have yet to be published or made available through materials obtained by taking the certification course. This assessment tool is marketed to coaches, strength and conditioning experts, athletic trainers, and physical therapists as a low-cost and portable clinical screen that could help identify and track progress of specific movement patterns that are key to achieving efficient pitching mechanics. ${ }^{14}$ However, it is unclear how this assessment tool can be used most appropriately because no studies regarding reliability of this screen exists. It has also yet to be studied if this screening tool has value in predicting injury risk or potentially injurious or inefficient pitching mechanics. Further, it is unknown if this screen should be used in place of or in conjunction with a pitching 3D motion analysis. The primary purpose of this study was to compare screen results from four OnBaseU tests to relevant pitching mechanics measured using 3D motion capture to evaluate if the OnBaseU screen can be used to assess pitching mechanics. The secondary purpose was to compare OnBaseU and 3D motion capture seated trunk rotation test results to determine the validity of the OnBaseU test. The authors hypothesized that OnBaseU tests would be associated with analogous pitching mechanics. The authors additionally hypothesized that On-

\begin{tabular}{c|ccc}
$\begin{array}{c}\text { Participant } \\
\text { Ages }\end{array}$ & $\begin{array}{c}\text { Number of } \\
\text { Participants }\end{array}$ & $\begin{array}{c}\text { Height } \\
(\mathbf{m} \text {; mean } \pm \text { SD) }\end{array}$ & $\begin{array}{c}\text { Weight } \\
(\mathbf{k g} ; \text { mean } \pm \text { SD) }\end{array}$ \\
\hline 13 years & $\mathrm{n}=10$ & $1.69 \pm 0.10$ & $57.43 \pm 9.17$ \\
\hline 14 years & $\mathrm{n}=27$ & $1.78 \pm 0.09$ & $73.04 \pm 15.17$ \\
\hline 15 years & $\mathrm{n}=18$ & $1.78 \pm 0.06$ & $76.15 \pm 7.73$ \\
\hline 16 years & $\mathrm{n}=32$ & $1.84 \pm 0.06$ & $80.82 \pm 9.85$ \\
\hline 17 years & $\mathrm{n}=12$ & $1.87 \pm 0.08$ & $88.34 \pm 13.79$ \\
\hline 18 years & $\mathrm{n}=3$ & $1.81 \pm 0.04$ & $72.73 \pm 4.91$ \\
\hline All & $\mathrm{n}=103$ & $1.80 \pm 0.09$ & $76.16 \pm 13.81$
\end{tabular}

\section{Table 1. Study Participant Demographics}

BaseU seated trunk rotation test results would be associated with 3D motion capture seated trunk rotation test results. The results of this study can be used to assess whether portions of the OnBaseU clinical movement screen analyzed in this study can be used to estimate corresponding pitching mechanics.

\section{METHODOLOGY}

\section{DATA COLLECTION}

One hundred and three adolescent pitchers (age $=15.2 \pm$ 1.29 years) completed the OnBaseU screening assessment and a 3D motion analysis of pitching. Participant demographics can be seen in Table 1. Participant inclusion criteria included youth baseball players ranging from ages 13 to 20 years who declared pitching as their primary position. No data were collected from players who were experiencing pain and player data were not included in this analysis if that participant did not complete both the OnBaseU screening and motion capture evaluation. After OnBaseU screening was completed, players were instructed to conduct a normal pre-game warmup prior to motion capture analysis. A motion capture seated trunk rotation test was also conducted on 80 of the 103 youth players (age $=15.2 \pm 1.32$ years; height $=1.80 \pm 0.0889 \mathrm{~m}$; weight $=75.7 \pm 13.9 \mathrm{~kg}$ ) . OnBaseU screening was performed by a strength and conditioning coach with over 12 years of experience who is certified by the College of Strength and Conditioning Coaches association (CSCCa) and OnBase University ${ }^{\circledR}$. Motion capture evaluations were conducted by a biomechanist who holds a doctorate degree with a research focus in motion capture biomechanics and sports medicine.

\section{ONBASEU DATA COLLECTION}

The screen consists of 16 consecutive tests intended to identify patterns in a player's pitching mechanics. For this study, four OnBaseU tests were selected for comparison by the strength and conditioning coach and biomechanist based on the face validity for what tests would be most directly associated with 3D motion capture metrics. These four tests were: (1) seated trunk rotation test, (2) side step walkout test, (3) push-off test, and (4) shoulder 90/90 test. The other 12 tests that are a part of the screening assessment are not analogous to pitching metrics and, therefore, were not included in comparisons for this study. Screening 
was conducted in accordance with OnBaseU methods. The screen was terminated if the participant communicated experiencing pain at any point during testing. Descriptions for how to conduct each of the four tests included in this study are detailed below.

A seated trunk rotation test has been described previously and has demonstrated inter-tester and intra-tester reliability when measured with a goniometer in healthy adults. ${ }^{15}$ OnBaseU adapted this test to include a cervical spine component and is measured using visual assessment, rather than a goniometer or other measurement device. The seated trunk rotation test (Figure 1A) measures cervicothoraco-lumbar spine mobility, which is postulated by OnBaseU to be associated with upper and lower body separation during a pitch. The test consists of two parts. First, the player is instructed to sit holding a bat across their shoulders, with their right foot crossed over the left. The player is then instructed to rotate their thorax to the right as far as possible without rotating the pelvis. OnBaseU grading criteria consists of a visual assessment of having completed a trunk rotation angle that is "greater than $45^{\circ}$ ", "equal to $45^{\circ}$ ", or "less than $45^{\circ}$ ". Second, the player is instructed to turn their head back to the left as far as possible while maintaining this rotated position. The player is evaluated according to OnBaseU grading criteria, which is a visual assessment of if they had turned their chin "over the clavicle" or "short of the clavicle". Both parts of the test are then performed and evaluated for the contralateral side.

The side step walkout test (Figure 2A) was developed by OnBaseU with the intention of identifying how hip and groin flexibility influences the player's ability to stride effectively during a pitch. To conduct this test, the player is first instructed to lay on the ground. Two baseballs are placed outside the foot and ipsilateral shoulder to serve as markers for the test. Next, the player is instructed to stand up, stand in-line with one baseball, and stride with the opposite foot as far as possible without losing balance. This is done for both left and right sides. OnBaseU criteria for evaluation consists of a visual assessment of whether the stride foot was "past the ball", "equal to the ball" or "less than the ball".

The push-off test (Figure 3A) was developed by OnBaseU in an effort to measure hip and groin flexibility during a stride motion, but it also combines these measures with lower body motor control evaluation. This test consists of two parts. First, the player is instructed to stride out as far as possible such that the striding foot lands perpendicular to the standing foot, similar to a stride performed when pitching. The distance of the stride is measured by the player's foot lengths. Foot length measurements, as defined by OnBaseU, measure distance as the number of lengths of the participants feet. This measurement is obtained by having the participant walk placing their heel against the contralateral toe and counting how many foot lengths were needed to cross the distance being measured. To the authors knowledge, this method of measuring distance has not been validated. OnBaseU grading criteria consists of a visual assessment of whether the distance between feet is "greater than 6 foot lengths", "5-6 foot lengths", or "less than 5 foot lengths". The second part of this test is conducted the same as the first, but in the second part the
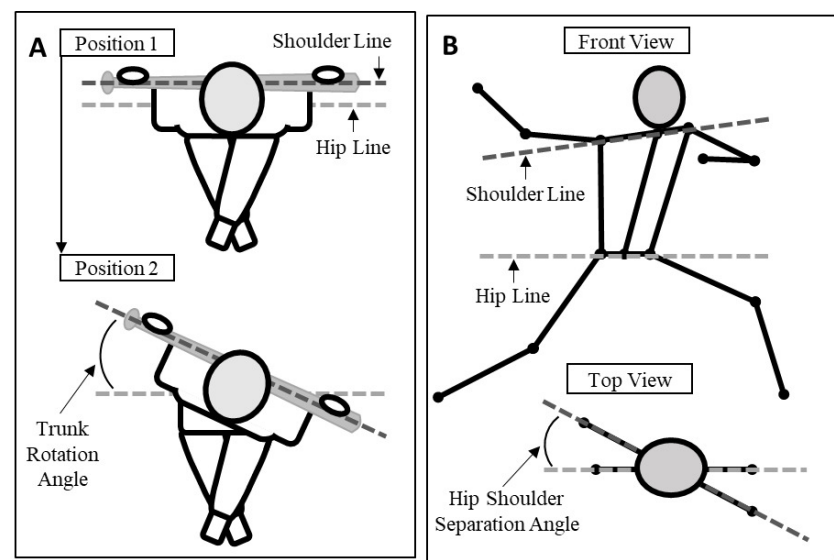

Figure 1. Schematic of A) OnBaseU seated trunk rotation test and $B$ ) hip shoulder separation at foot strike motion capture metric.
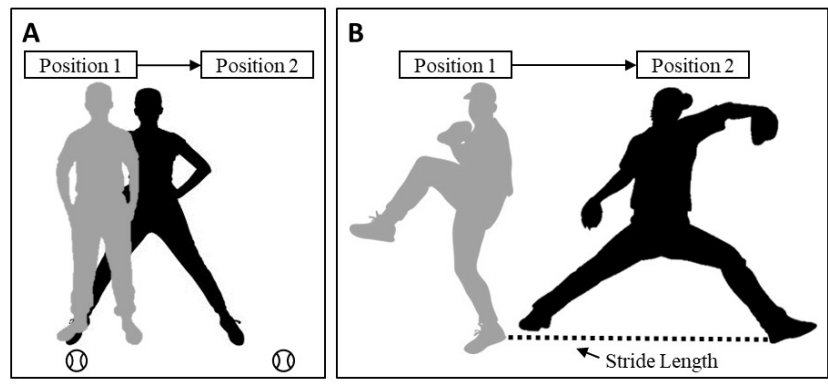

Figure 2. Schematic of A) OnBaseU side step walkout test and $B$ ) stride length motion capture metric.
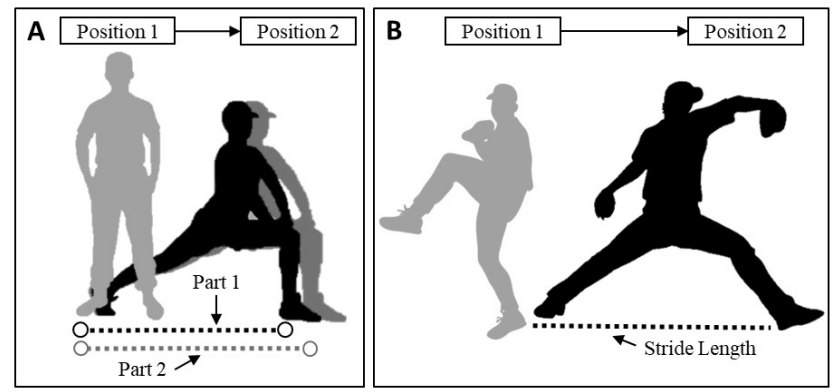

Figure 3. Schematic of A) OnBaseU push-off test parts 1 and 2 and $B$ ) stride length motion capture metric.

player is instructed to drive off the standing leg and allow that foot to drag as they would in a normal pitch. OnBaseU evaluation for this part consists of a visual assessment of if the additional distance between the feet was "greater than 1 foot length", “0.5-1 foot lengths", or "less than 0.5 foot lengths" of additional distance from part 1 . This was done for both left and right sides.

The shoulder 90/90 test (Figure 4A) was previously developed $^{16}$ and was included in this screen with the intention of testing gleno-humeral joint mobility and scapulo-thoracic 
stability, according to OnBaseU. For this test, the player is instructed to stand with slight hip and knee flexion and hold the arm out with $90^{\circ}$ elbow flexion and $90^{\circ}$ shoulder abduction. In this position, the player is then told to externally rotate at the shoulder joint as far as possible without spinal extension. This is performed and evaluated for both arms. OnBaseU grading criteria evaluates movement by visually assessing if the forearm long-axis rotation about the shoulder joint was "greater than the spinal angle", "equal to the spinal angle", or "less than the spinal angle".

\section{MOTION CAPTURE DATA COLLECTION}

Motion capture data were collected at $250 \mathrm{~Hz}$ using the 40 reflective marker set required for PitchTrak (Motion Analysis Corporation, Santa Rosa, California) and a twelve-camera motion analysis system (Qualisys AB, Göteborg, Sweden). The mound was engineered to meet major league specification, and pitches were thrown to a catcher located at major league regulation distance. Participants conducted a normal pre-game warmup, followed by pitching four fastballs, four changeups, and four breaking balls. Reports of kinetic and kinematic data were generated using Visual3D (C-Motion, Inc, Germantown, MD) and the Qualisys Baseball PAF (Qualisys AB, Göteborg, Sweden). Only data from fastballs were included in this study. These metrics were hip shoulder separation at foot strike $\left({ }^{\circ}\right)$ (Figure 1B), stride length (\%body-height) (Figure $\underline{2 B}$ and $\underline{3 B}$ ), and shoulder maximum external rotation $\left({ }^{\circ}\right.$ ) (Figure $4 \mathrm{~B}$ ). Hip shoulder separation at foot strike is the angle between the axis created by the left and right acromion markers and the axis created by the left and right anterior superior iliac spine markers. Shoulder external rotation is the rotation about the long-axis of the humerus.

In addition to metrics calculated from pitching evaluations, trunk rotation during a seated trunk rotation test was measured using the motion capture system. Players were instructed to sit with their arms crossed across their chest such that their hands were placed on their shoulders and a soccer ball was placed between their knees. They were then told to rotate their thorax as far as possible without rotating the pelvis. Trunk rotation was the angle between the axis created from the right and left shoulder acromion markers and the axis created from the right and left anterior superior iliac spine.

\section{STATISTICAL ANALYSES}

Only OnBaseU screen data from the throwing side were included in this study as these data were relevant for comparison with pitching biomechanics 3D motion capture data. For quantitative analysis in this study, OnBaseU scoring categories were converted to point values. This was done for the two-part tests to be condensed into one score that could be used for statistical analysis. Tests that only contained one part were also converted to point values for consistency. Points were assigned to the evaluations where the best to worst test performance was awarded the greatest to least number of points. For part one of the OnBaseU seated trunk rotation test, evaluations of "greater than $45^{\circ}$ " were assigned three points, “equal to $45^{\circ}$ ” were assigned
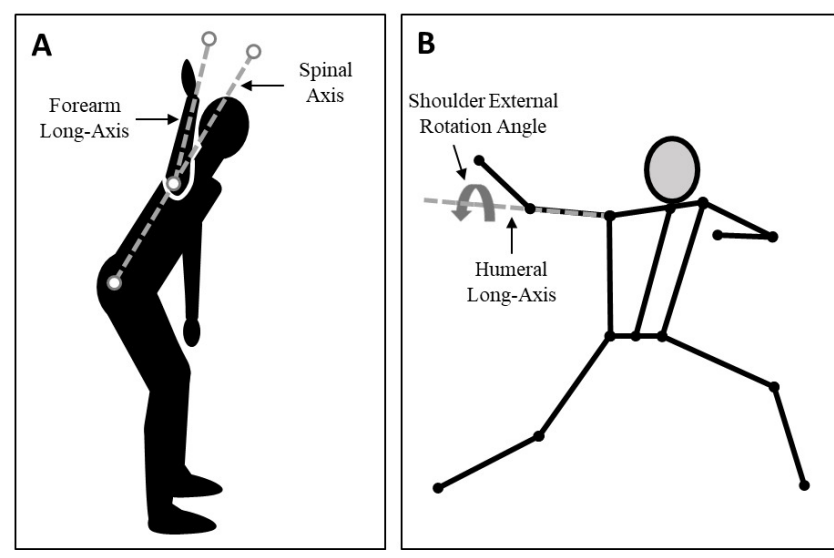

Figure 4. Schematic of A) OnBaseU shoulder 90/90 test and $B$ ) shoulder maximum external rotation motion capture metric.

two points, and "less than $45^{\circ}$ " were assigned one point. For part two of this test, evaluations of "over the clavicle" were assigned two points and "short of the clavicle" were assigned one point. Points from the two parts were combined to create one test grade ranging from 2-5. For the side-step walkout test data, evaluations of "past the ball" were assigned a test grade of 3 , "equal to the ball” were assigned a grade of 2 , and "less than the ball" were assigned a grade of 1 . For part one of the push-off test, evaluations of " $>6$ foot lengths" were assigned three points, "5-6 foot lengths" were assigned two points, and " $<5$ foot lengths" were assigned one point. For the second part of this test, evaluations of ">1 foot length gain" were assigned three points, "0.5-1 foot length gain" were assigned two points, and " $<0.5$ foot length gain" were assigned one point. Points from both parts of the test were combined to create one test grade ranging from 2-6. For the shoulder 90/90 test, evaluations of "greater than spinal angle" were assigned a test grade of 3, "equal to spinal angle" were assigned a test grade of 2 , and "less than spinal angle" were a grade of 1 .

Correlation coefficients and corresponding p-values and 95\% confidence intervals were calculated to determine if OnBaseU tests were associated with pitching metrics that were hypothesized to be related. Correlation coefficients of $r<0.1,0.1 \leqslant r<0.3,0.3 \leqslant r<0.5$, and $r \geqslant 0.5$ were defined as not correlated, minorly correlated, moderately correlated and highly correlated, which is in accordance with Cohen's definition. ${ }^{17}$ Additionally, average pitching mechanics from different OnBaseU scoring categories were compared to understand if OnBaseU scoring criteria possessed precision such that different scoring categories result in different mechanics. If statistical differences were not found between groups, this would indicate that players with similar pitching mechanics could obtain different scores on the OnBaseU test, and that scoring criteria as it is currently defined is potentially not useful. Visual inspection of the box and whisker plots of the four comparisons showed that the data were not normally distributed. Therefore, a non-parametric Kruskall-Wallis test with a Chi-squared approximation was performed using JMP (JMP, Cary, NC) to test if there were differences between averages of any groups. If the two- 
tailed p-value from the Kruskall-Wallis test was significant $(p<0.05)$, a Dunn's post-hoc test was used to assess which pairwise comparisons might be significant. A Common Language Effect Size (CLES) and 95\% confidence interval (CI) for that effect size was reported for each comparison that yielded a statistically significant p-value from the Dunn's post-hoc test. The CLES modifies the $\mathrm{d}_{\text {Cohen }}$ effect size by calculating the pooled standard deviation with weights to account for groups with different sample sizes.

\section{RESULTS}

Motion capture metrics and OnBaseU screen results from 103 adolescent baseball pitchers were compared. Figure 5A$\underline{D}$ shows comparisons between motion capture metrics ( $\mathrm{y}-$ axis) and the OnBaseU tests ( $\mathrm{x}$-axis) that were hypothesized to be associated (to discern face validity).

Hip shoulder separation at foot strike $\left(^{\circ}\right)$ and OnBaseU seated trunk rotation test data (Figure 5A) were minorly correlated $(r=0.19 ; p=0.05 ; 95 \% \mathrm{CI}=-0.003-0.37)$. Different OnBaseU seated trunk rotation test grades did not correspond to statistically significantly different hip shoulder separation at foot strike angles $\left({ }^{\circ}\right)$ from the motion capture system $(p=0.18)$.

Stride length during pitching (\%body-height) and OnBaseU side step walkout test data (Figure 5B) were moderately correlated $(r=0.34 ; p=0.0003 ; 95 \% \mathrm{CI}=0.16-0.50)$. Stride lengths (\% body-height) from the motion capture system of players who obtained OnBaseU side step walkout test grades of 1 vs. 3 were statistically significantly different $(p<0.01 ;$ CLES $=0.735 ; \mathrm{CI}=0.395-1.378)$. Comparisons of all other groups in this dataset were not significantly different.

Stride length (\% body-height) during pitching and OnBaseU push-off test data (Figure 5C) were not correlated ( $\mathrm{r}$ $=0.18 ; \mathrm{p}=0.06 ; 95 \% \mathrm{CI}=-0.01-0.36)$. Different OnBaseU push-off test scores did not correspond to statistically significantly different stride length data (\% body-height) from the motion capture system $(\mathrm{p}=0.07)$.

Shoulder maximum external rotation $\left({ }^{\circ}\right)$ and the shoulder 90/90 test data (Figure 5D) were not correlated $(r=0.13$; $\mathrm{p}=0.18 ; 95 \% \mathrm{CI}=-0.06-0.32$ ). Different OnBaseU shoulder $90 / 90$ scores did not correspond to statistically significantly different shoulder maximum external rotation data $\left(^{\circ}\right)$ from the motion capture system $(\mathrm{p}=0.10)$.

OnBaseU and motion capture seated trunk rotation test data (igure 6) were not correlated $(r=0.003 ; p=0.98 ; 95 \%$ $\mathrm{CI}=-0.22-0.22$ ). OnBaseU seated trunk rotation scores did not correspond to motion capture seated trunk rotation test data $(\mathrm{p}=0.83)$.

\section{DISCUSSION}

The hypothesis that OnBaseU tests would be associated with analogous pitching mechanics was not supported by the results of this study. The four comparisons between pitching mechanics and OnBaseU tests analyzed in this report had an overall positive trend (Figure 5A-D). Only stride length (\%body-height) during pitching and the side step walkout test results were moderately correlated, while all
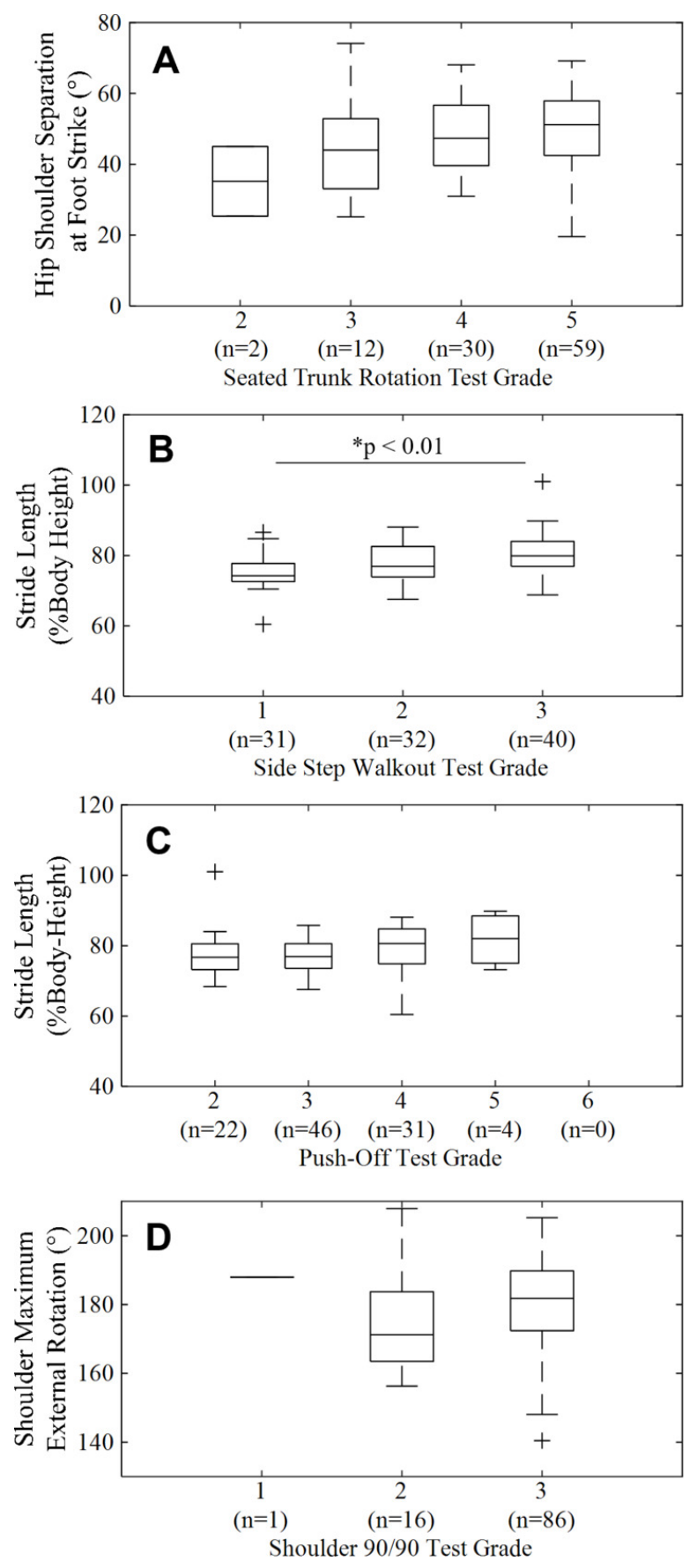

Figure 5. Box and whisker plots display data range.

The box shows the 25 th to 75 th percentile, the line across the interior is the median, and the whiskers display the minimum and maximum. The "+" symbol labels outliers. (A) Comparison of hip shoulder separation at foot strike $\left(^{\circ}\right)$ and OnBaseU seated trunk rotation test performance. (B) Comparison of stride length (\% body-height) and OnBaseU side step walkout test performance. (C) Comparison of stride length (\% body-height) and OnBaseU push-off test performance. (D) Comparison of shoulder maximum external rotation $\left({ }^{\circ}\right.$ ) and OnBaseU shoulder 90/90 test performance. P-values from Wilcoxon non-parametric tests are shown above statistically significant $(\mathrm{p}<0.05)$ comparisons.

other comparisons between OnBaseU tests and pitching mechanics were either minorly correlated or not correlated. Further, only the stride length (\%body-height) of individuals who scored a 1 and 3 in the OnBaseU side step walkout test was statistically significantly different $(\mathrm{p}<0.01)$. Contrary to the secondary hypothesis, OnBaseU seated trunk rotation test data and the motion capture seated trunk rota- 
tion test data were not correlated, calling into question the validity of the visual scoring method and evaluation criteria in the OnBaseU screen (Figure 6). While most OnBaseU tests were not found to be associated with 3D motion capture pitching mechanics examined in this study, they may have value when used in other contexts to identify areas of deficiency that may lead to improper mechanics. Further study is required to understand how this movement screen can be used.

In the OnBaseU literature, the seated trunk rotation test adapted by OnBaseU is described as a measurement of trunk axial flexibility. According to Senington et al., hip-shoulder separation is an accurate measurement of trunk axial flexibility, particularly lateral flexion, ${ }^{18}$ making it a reasonable comparison to the OnBaseU seated trunk rotation screen. However, hip shoulder separation during throwing is a dynamic movement that is affected by not only flexibility, but also strength, timing, and skill. The OnBaseU seated trunk rotation test was unable to estimate hip shoulder separation at foot strike, but if used in combination with a motion capture evaluation, it could potentially inform regarding why a pitcher may demonstrate poor hip shoulder separation by either identifying or eliminating axial flexibility as a cause.

The OnBaseU seated trunk rotation test score results were also compared to a seated trunk rotation test measured using motion capture. OnBaseU seated trunk rotation test grades did not demonstrate statistically significant differences when compared with motion capture seated trunk rotation results. OnBaseU scoring criteria may lack the precision to distinguish between trunk rotation abilities through visual analysis by the evaluator. This calls into question the validity of the scoring criteria and if it is defined in a way that the test can be conducted with accuracy. Seated trunk rotation angle was an average of $62.8^{\circ} \pm 10.1^{\circ}$ for all 80 pitchers. This is comparable to previous work that reported an average seated trunk rotation angle of $69.9^{\circ} \pm$ $9.8^{\circ}$ in 21 non-injured collegiate baseball players. ${ }^{19}$

The side step walkout test is described by OnBaseU as a measurement of hip and groin flexibility, and since stride length during a pitch has been shown to be associated with bilateral total arc of adduction and abduction of the hips, ${ }^{20}$ these two measurements were compared. Those who scored a 1 and 3 on the OnBaseU side step walkout test had statistically significantly different stride lengths during pitching $(p<0.01)$. This test may, therefore, have value in predicting stride length. When used in conjunction with motion analysis, the side step walkout test could potentially inform whether hip and groin flexibility is either too low or high in players with undesirable stride lengths, thereby aiding coaches and athletic trainers in developing a program for that player. A comparison of passive hip range of motion and the side step walkout test is needed to further validate this test.

In addition to the side step walkout test, OnBaseU uses the push-off test to evaluate hip and groin flexibility during a stride motion as well. Therefore, these test results were also compared to stride length during pitching measured using 3D motion capture. The push-off test was not associated with stride length. The recommended stride length is approximately $80-90 \%$ of body-height in adult pitchers.

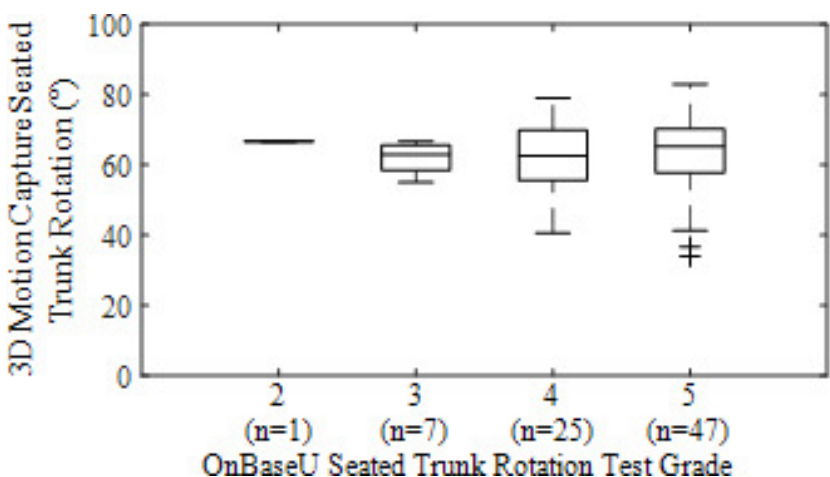

Figure 6. Box and whisker plot comparison of OnBaseU seated trunk rotation test and seated trunk rotation test measured by a motion capture system $\left({ }^{\circ}\right)$.

Smaller stride lengths of approximately $50 \%$ body-height have been shown to increase throwing arm momentum, potentially increasing injury risk in adults. ${ }^{21}$ In youth athletes, every $10 \%$ increase in stride length has been found to be correlated with a $1.9 \pm 0.4$ mile per hour pitch velocity increase. ${ }^{22}$ Larger stride lengths alter timing of stride foot contact, shortening double support phase of the pitch. This may result in loss of momentum transferred through the kinematic chain. ${ }^{23}$ However, larger stride lengths of up to $100 \%$ body-height are sometimes beneficial and have been found to be associated with increased ball velocity. ${ }^{24}$ In the current study, most pitchers had stride lengths within the recommended range and the sample size of players who scored a 5 or 6 was very low $(n=0-4)$. This raises the question of whether the higher 5 or 6 test scores are achievable, would be considered over-striding, and whether this stride length would be advantageous for that player.

The shoulder 90/90 test was adapted by OnBaseU to highlight any limitations in glenohumeral joint range of motion and/or stability of the scapula-thoracic junction. This test was compared to maximum external rotation of the throwing shoulder during the late cocking phase of a pitch measured using 3D motion capture as this metric has been shown to represent gleno-humeral joint mobility as well as scapular and thoracic movements during a pitch. ${ }^{25}$ The shoulder 90/90 test scores of 2 and 3 were not associated with maximum shoulder external rotation angles obtained using motion capture during pitching. However, if used in conjunction with motion analysis, the OnBaseU test may inform whether flexibility is the reason for disadvantageous maximum external rotation.

A potential reason for the lack of association between OnBaseU tests and pitching mechanics is that OnBaseU is a series of movement tests that are performed prior to warmup. Warmup is a key aspect of athletic performance that has been shown to increase flexibility, providing benefits such as improved joint range of motion as well as enhanced muscular performance. ${ }^{26}$ Therefore, since OnBaseU screens are performed prior to warmup, these aspects of the pitching motion may vary from when they are evaluated by the motion capture system post-warmup. Additionally, most OnBaseU tests are static and isolate a single area of 
interest to be measured. Though this may be effective for identifying specific physical limitations, it does not accurately simulate the dynamic and complex pitching motion. Also, many OnBaseU tests measure an athlete's active range of motion, rather than the dynamic measurements that are displayed during a real-time pitching motion. In throwing athletes, dynamic range of external shoulder rotation is significantly higher than active or passive range of motion measurements due to active muscle tone ${ }^{27}$ so it is reasonable to infer that similar effects may be observed in aspects of the pitching motion as well.

In addition to biomechanical differences between pitching evaluation methods, sample size could have affected this analysis. The side step walkout test was the only OnBaseU test that had enough participants in the two most extreme categories (i.e. test score of 1 vs. test score of 3 ) to be able to perform a statistical comparison. All other OnBaseU tests lack the sample size in either the lowest or highest test scores $(n=0-4)$ to allow for reasonable comparison of those categories (Figure 5A-D). Given that so few participants achieved these scores out of 103 players, it is unclear if these scores are physiologically relevant or even reasonably achievable. These results call into question how the intermediate scoring categories can be used to assess efficient or deficient movement patterns specific to pitching. It can be seen that intermediate scoring categories lack the precision to be associated with corresponding pitching mechanics. Similarly, the seated trunk rotation test measured using motion capture revealed that the OnBaseU seated trunk rotation test scoring criteria may not defined in a way that can be used correctly. Further study is required to determine what ranges of motion can be visually distinguished from each other for each test and scoring criteria should be adjusted accordingly. Additional study should also include multiple evaluators to exclude the variable of evaluator error. An additional question is whether the OnBaseU seated trunk rotation, push-off and shoulder 90/90 test lowest and highest test score categories can predict corresponding pitching mechanics collected with a motion capture system. If this were found to be the case, however, it must be noted that the predictive value of those two OnBaseU scoring categories would only be in forecasting binary "good" vs. "bad" mechanics.

It is important to acknowledge the limitations of this study. Further analysis is needed to examine predictive potential of the lowest and highest test grades from the OnBaseU seated trunk rotation test, push-off test and shoulder 90/90 tests. Few athletes fell into either the lowest or highest test score categories in these three tests, so comparison of these groups was either not possible or not reasonable. However, given how few players received these scores out of 103 participants, it is unlikely that the addition of more participants would increase the sample size in these scoring categories and may indicate that these scores are not relevant or not reasonably achievable. Another limitation of this study is that each part of a two-part test was weighted equally when combining them in order to create a quantifiable score for analysis. Therefore, if one part of the test was more clinically significant than the other, this was not reflected in the analysis. However, consensus among those trained in the use of the OnBaseU screen is that the two parts combined in this study were of equal clinical significance. The OnBaseU seated trunk rotation test was completed with different methods than the motion capture seated trunk rotation test. The OnBaseU test included a second part to the test that evaluated cervical spine motion while the motion capture test did not. It is possible that the lack of correlation between the OnBaseU and motion capture seated trunk rotation tests is due to the differences in how the tests were conducted. While the age range of 14-20 years included players of varying skill, height and weight, statistical analysis of data obtained from 13-15 year-olds, 16-18 year-olds and 19-20 year-olds found that OnBaseU and motion capture data had no statistically significant relationships. These data were not included in this manuscript as they elicited no additional information beyond data presented here. Finally, it is unclear what the effect of education and experience of the evaluator had on the results of the OnBaseU screen. This study included data produced by only one evaluator so no additional variability from multiple evaluators were introduced. However, prior study of the Selective Functional Movement Screen ${ }^{\mathrm{TM}}$ found that experience as an evaluator did affect intra- and interrater reliability using a visual and subjective scoring method. ${ }^{28}$ Considering that there are no education requirements to obtain certification to administer this screen, evaluators will have varying training and knowledge that could alter reliability of the screen.

\section{CONCLUSION}

OnBaseU seated trunk rotation, push-off and shoulder 90/ 90 tests were found to demonstrate either minor correlations or not be correlated with corresponding 3D motion capture measurements of pitching mechanics. Only the stride length (\% body-height) during pitching and side step walkout test data were moderately correlated. Stride lengths (\% body-height) of players who scored a 1 or 3 on the OnBaseU side step walkout test groups were statistically significantly different $(\mathrm{p}<0.01)$. Therefore, these two side step walkout test scoring categories may be able to provide information about stride length during pitching. Statistically different results between OnBaseU and motion capture measures of the seated trunk rotation tests indicate that the OnBaseU seated trunk rotation test may not accurately evaluate trunk rotation. The results of this study indicate that it may not be appropriate to assess youth pitching mechanics with the OnBaseU clinical assessment screen. Future study comparing OnBaseU results to metrics of movement, pain, and injury are needed to determine the value of this screen.

\section{CONFLICTS OF INTEREST}

The authors have no conflicts of interest to disclose.

\section{FUNDING}

The work presented in this study was supported by departmental funding. 
Submitted: November 19, 2020 CST, Accepted: September 04, 2021 CST

This is an open-access article distributed under the terms of the Creative Commons Attribution 4.0 International License (CCBY-NC-4.0). View this license's legal deed at https://creativecommons.org/licenses/by-nc/4.0 and legal code at https://creativecommons.org/licenses/by-nc/4.0/legalcode for more information. 


\section{REFERENCES}

1. Fleisig GS, Andrews JR. Prevention of Elbow Injuries in Youth Baseball Pitchers. Sports Health. 2012;4(5):419-424. doi:10.1177/1941738112454828

2. Fleisig GS, Andrews JR, Cutter GR, et al. Risk of serious injury for young baseball pitchers: A 10-year prospective study. Am J Sports Med. 2011;39(2):253-257. doi:10.1177/0363546510384224

3. Fortenbaugh D, Fleisig GS, Andrews JR. Baseball pitching biomechanics in relation to injury risk and performance. Sports Health. 2009;1(4):314-320. doi:1 $\underline{0.1177 / 1941738109338546}$

4. Chalmers PN, Wimmer MA, Verma NN, et al. The relationship between pitching mechanics and injury: A review of current concepts. Sports Health. 2017;9(3):216-221. doi:10.1177/1941738116686545

5. Anz AW, Bushnell BD, Griffin LP, Noonan TJ, Torry MR, Hawkins RJ. Correlation of torque and elbow injury in professional baseball pitchers. Am J Sports Med. 2010;38(7):1368-1374. doi:10.1177/0363546510 $\underline{363402}$

6. Aguinaldo AL, Buttermore J, Chambers H. Effects of upper trunk rotation on shoulder joint torque among baseball pitchers of various levels. J Appl Biomech. 2007;23(1):42-51. doi:10.1123/jab.23.1.42

7. Gould ZI, Oliver JL, Lloyd RS, Neil R, Bull M. The Golf Movement Screen is related to spine control and $\mathrm{X}$-factor of the golf swing in low handicap golfers. $J$ Strength Cond Res. July 2018:1. doi:10.1519/jsc.00000 $\underline{00000002664}$

8. Kiesel K, Plisky PJ, Voight ML. Can Serious Injury in Professional Football be Predicted by a Preseason Functional Movement Screen? N Am J Sports Phys Ther. 2007;2(3):147-158.

9. Bonazza NA, Smuin D, Onks CA, Silvis ML, Dhawan A. Reliability, validity, and injury predictive value of the Functional Movement Screen. Am J Sports Med. 2017;45(3):725-732. doi:10.1177/0363546516641937

10. Cook G, Burton L, Hoogenboom B. Preparticipation screening: the use of fundamental movements as an assessment of function - part $1 . N$ Am J Sports Phys Ther. 2006;1(2):62-72.

11. Smith CA, Chimera NJ, Wright NJ, Warren M. Interrater and intrarater reliability of the functional movement screen. J Strength Cond Res. 2013;27(4):982-987. doi:10.1519/JSC.0b013e3182606d f2
12. Chorba RS, Chorba DJ, Bouillon LE, Overmyer CA, Landis JA. Use of a functional movement screening tool to determine injury risk in female collegiate athletes. N Am J Sports Phys Ther. 2010;5(2):47-54.

13. O’Connor FG, Deuster PA, Davis J, Pappas CG, Knapik JJ. Functional movement screening: Predicting injuries in officer candidates. Med Sci Sports Exerc. 2011;43(12):2224-2230. doi:10.1249/MS S.0b013e318223522d

14. OnBaseU - OnBaseU.com. http://www.onbaseu.co m/. Accessed May 25, 2021.

15. Johnson KD, Kim K-M, Yu B-K, Saliba SA, Grindstaff TL. Reliability of thoracic spine rotation range-of-motion measurements in healthy adults. $J$ Athl Train. 2012;47(1):52-60. doi:10.4085/1062-605 $\underline{0-47.1 .52}$

16. Speariett S, Armstrong R. The relationship between the Golf-Specific Movement Screen and golf performance. J Sport Rehabil. 2019;29(4):425-435. do i:10.1123/JSR.2018-0441

17. Cohen J. A Power Primer. Psychol Bull. 1992;112:155.

18. Senington B, Lee RY, Williams JM. Are shoulder counter rotation and hip shoulder separation angle representative metrics of three-dimensional spinal kinematics in cricket fast bowling? J Sports Sci. 2018;36(15):1763-1767. doi:10.1080/02640414.2017.1 $\underline{416734}$

19. Chasse P, Bullock GS, Schmitt AC, Little BA, Diehl LH, Butler RJ. The relationship between trunk rotation, upper quartter dynamic stability, and the Kerlan-Jobe orthopaedic clinic overhead athete shoulder and elbow score in Division 1 collegiate pitchers. Int J Sports Phys Ther. 2018;13(5):819.

20. Robb AJ, Fleisig G, Wilk K, MacRina L, Bolt B, Pajaczkowski J. Passive ranges of motion of the hips and their relationship with pitching biomechanics and ball velocity in professional baseball pitchers. $\mathrm{Am}$ J Sports Med. 2010;38(12):2487-2493. doi:10.1177/036 $\underline{3546510375535}$

21. Ramsey DK, Crotin RL, White S. Effect of stride length on overarm throwing delivery: A linear momentum response. Hum Mov Sci. 2014;38:185-196. doi:10.1016/j.humov.2014.08.012 
22. Sgroi T, Chalmers PN, Riff AJ, et al. Predictors of throwing velocity in youth and adolescent pitchers. $J$ Shoulder Elb Surg. 2015;24(9):1339-1345. doi:10.1016/ j.jse.2015.02.015

23. Crotin RL, Bhan S, Ramsey DK. An inferential investigation into how stride length influences temporal parameters within the baseball pitching delivery. Hum Mov Sci. 2015;41:127-135. doi:10.1016/ j.humov.2015.03.005

24. Montgomery J, Knudson D. A method to determine the stride length for baseball pitching. Appl Res Coach Athl. 2002;17:75-84.

25. Miyashita K, Kobayashi H, Koshida S, Urabe Y. Glenohumeral, scapular, and thoracic angles at maximum shoulder external rotation in throwing. Am J Sports Med. 2010;38(2):363-368. doi:10.1177/036354 6509347542
26. Pollock ML, Gaesser GA, Butcher JD, et al. The recommended quantity and quality of exercise for developing and maintaining cardiorespiratory and muscular fitness, and flexibility in healthy adults. Med Sci Sports Exerc. 1998;30(6):975-991. doi:10.109 7/00005768-199806000-00032

27. van den Tillaar R. Comparison of range of motion tests with throwing kinematics in elite team handball players. J Sports Sci. 2016;34(20):1976-1982. doi:10.10 $\underline{80 / 02640414.2016 .1149601}$

28. Stanek JM, Smith J, Petrie J. Intra- and inter-rater reliability of the Selective Functional Movement Assessment (SFMA) in healthy participants. Int J Sports Phys Ther. 2019;14(1):107-116. http://www.ncb i.nlm.nih.gov/pubmed/30746297. 